\title{
Automorphisms of Riemann surfaces with two fixed points
}

\author{
by Tomasz Szemberg (Kraków)
}

\begin{abstract}
We give an upper bound for the order of an automorphism of a Riemann surface with two fixed points. The main results are presented in Theorems 1.4 and 2.4.
\end{abstract}

0. Introduction. We assume the reader to be familiar with basic concepts in the theory of Riemann surfaces. Notions of hyperbolicity, genus, automorphism, quotient space etc. are used freely without further comments. Precise definitions may be found in [F-K]. As far as possible we also stick to the notation of that book.

The study of Riemann surfaces via their mappings is an old story. It is surprising how much information about the surface itself is contained in the properties of its mappings. The first important observation concerning the whole group of automorphisms of a hyperbolic, compact Riemann surface is contained in the Hurwitz theorem (see Theorem 1.1). The cyclic subgroups of that group were studied by Wiman, Kodaira and Lewittes. Their results were completed by Harvey (see Theorem 1.2).

It was Minda who noticed that all self-mappings of a hyperbolic Riemann surface with two fixed points must be automorphisms. This is not true in the non-hyperbolic case; fortunately there are only few such surfaces. Recently Jarnicki and Tworzewski [J-T] got a much sharper theorem (see Theorem $0.1)$.

Let $X$ be a Riemann surface. The group of its automorphisms is denoted by $\operatorname{Aut}(X)$. If $a, b$ are two distinct points of $X$, we denote by $G(X ; a, b)$ the set of all self-mappings of $X$ with $a$ and $b$ fixed.

Theorem 0.1. If $X$ is a hyperbolic Riemann surface then $G(X ; a, b)$ is a finite, cyclic subgroup of $\operatorname{Aut}(X)$.

It seems natural to ask what the order of the above group can be. As 
different techniques are involved we deal separately with compact surfaces and with domains in the complex plane.

1. Compact surfaces. Throughout this section $M$ denotes a compact Riemann surface of genus $g$. We assume that $g \geq 2$, that is, the surface is hyperbolic. Further, $T$ is an automorphism of $M$ with $\nu(T)$ fixed points. The subgroup of $\operatorname{Aut}(M)$ generated by $T$ is denoted by $\langle T\rangle$. If $G$ is any group we denote the number of its elements by ord $G$. Instead of $\operatorname{ord}\langle T\rangle$ we simply write ord $T$.

We look for a bound on the order of $T$ in terms of the genus $g$ of the surface on which it operates. This seems very natural in view of the following two statements.

Theorem 1.1 (Hurwitz 1893). ord Aut $(M) \leq 84(g-1)$.

Theorem 1.2 (Harvey, $[\mathrm{H}]$ 1966). For any $T \in \operatorname{Aut}(M)$, ord $T \leq 4 g+2$.

For automorphisms with one fixed point the above theorem is sharp:

EXAMPLE 1.3. Let $M$ be the normalization of the projective closure of a curve given by

$$
w^{2}=z\left(z^{2 g+1}-1\right), \quad g \geq 2 .
$$

Thus $M$ is a hyperelliptic Riemann surface of genus $g$.

Let $T(z, w)=\left(\varepsilon_{2 g+1} z, \varepsilon_{4 g+2} w\right)$, where $\varepsilon_{p}$ denotes a $p$-primitive root of 1. It is easily seen that $T \in \operatorname{Aut}(M)$ and $(0,0)$ is its fixed point. Of course ord $T=4 g+2$.

In case of two fixed points Harvey's result can be sharpened:

TheOREM 1.4. If $\nu(T) \geq 2$ then ord $T \leq 4 g$.

Proof. Let $M^{*}$ be the quotient Riemann surface $M /\langle T\rangle, \gamma$ its genus and $N=\operatorname{ord} T$. Let $\pi: M \rightarrow M^{*}$ be the quotient map. $\pi$ is thus an $N$-sheeted branch covering. We apply the Riemann-Hurwitz formula to $\pi$ to get

$$
2 g-2=N(2 \gamma-2)+B
$$

where $B$ denotes the branch index of $\pi$.

Case 1: either $\nu(T)>2$ or $\gamma>0$. The branch index of the quotient map at fixed points of $T$ is $N-1$, thus $B \geq \nu(T)(N-1)$. Applying this in (*) we get $2 g-2 \geq N[2 \gamma+\nu(T)-2]+\nu(T)(N-1)$. Since the expression in square brackets is not zero we get immediately

$$
N \leq \frac{2 g+\nu(T)-2}{2 \gamma+\nu(T)-2} \leq 2 g+1 \leq 4 g .
$$

Case 2: $\nu(T)=2$ and $\gamma=0$. From the Riemann-Hurwitz formula it follows at once that $T$ has at least one orbit of order $p$, where $1<p<$ 
$N$ (otherwise $g=0$, which contradicts the hyperbolicity of $M$ ). From elementary group theory it follows that $p$ must divide $N$ and thus $p \leq N / 2$. This time the branch index $B$ satisfies

$$
B \geq 2(N-1)+p(N / p-1)=3 N-2-p .
$$

Once again we link this with $(*)$ to get $2 g-2 \geq-2 N+3 N-2-p$ and consequently $N \leq 2 g+p \leq 2 g+N / 2$, which yields the desired $N \leq 4 g$.

Remark 1.5. Although our result is close to that of Harvey we got it using much simpler methods. In fact, only an elementary formula in the theory of compact Riemann surfaces (the Riemann-Hurwitz formula) was applied.

Remark 1.6. The estimate of Theorem 1.4 is sharp, that is, for any $g \geq 2$ there exists a compact Riemann surface $M_{g}$ of genus $g$ and points $a, b \in M_{g}$ such that there exists an automorphism $T \in G(M ; a, b)$ with $\operatorname{ord} T=4 g$.

ExAmPLE 1.7. Let $M_{g}$ be the normalization of the projective closure of the curve given by

$$
w^{2}=z\left(z^{2 g}-1\right), \quad g \geq 2 .
$$

$M_{g}$ is a hyperelliptic compact Riemann surface of genus $g$. Let $T(z, w)=$ $\left(\varepsilon_{2 g} z, \varepsilon_{4 g} w\right)$, where $\varepsilon_{p}$ is as in Example 1.3. It is easily seen that $T \in \operatorname{Aut}(M)$ and $(0,0)$ and the point at infinity are its fixed points. Moreover, ord $T=4 g$.

2. Domains in complex plane. The key concept in this part is that of "complex double". We sketch briefly the construction of this surface. The first step is to notice that any domain may be conformally mapped onto another one of a particularly nice type. Throughout the section $\Omega$ denotes an open domain in the complex plane, $\partial \Omega$ its boundary and $\bar{\Omega}$ its closure.

DEFinition 2.1. Domain whose border consists of $p$ circles, some of them possibly being points, is call p-circular. If all components of its boundary are circles with positive radius we call the domain proper.

In the sense of the above definition annuli are 2-circular proper domains. Circular domains are canonical as the following theorem shows.

TheOREM 2.2 (Goluzin [G]). Let $\Omega$ be a p-connected bounded domain in the complex plane. Then there exists a bounded p-circular domain $\Delta$ and $a$ conformal map $s: \Omega \rightarrow \Delta$.

Of course $s$ induces an isomorphism of automorphism groups,

$$
\operatorname{Aut}(\Omega) \ni g \rightarrow s \circ g \circ s^{-1} \in \operatorname{Aut}(\Delta) .
$$

If $\Omega$ is a circular domain and $T \in \operatorname{Aut}(\Omega)$ then by the Riemann theorem on isolated singularities $T$ may be extended through the isolated points of 
$\partial \Omega$. So it suffices to consider automorphisms of proper circular domains. Moreover, the following holds.

Theorem 2.3 (Behnke-Sommer [B-S]). Let $\Omega$ be a proper p-circular domain and let $T \in \operatorname{Aut}(\Omega)$. Then $T$ may be extended to a homeomorphism $\widetilde{T}$ of $\bar{\Omega}$.

After these preparations we can construct the "complex double" (cf. [B-S]). Let $\Omega$ be a bounded proper $p$-circular domain. Let $\Omega^{+}$and $\Omega^{-}$ be two copies of $\Omega$. We assume that $\Omega^{+}$has the usual complex structure and the other copy the conjugate one. If we glue $\bar{\Omega}^{+}$and $\bar{\Omega}^{-}$together by identifying the corresponding points of $\partial \Omega^{+}$and $\partial \Omega^{-}$the resulting compact manifold $M$ clearly has genus $g=p-1$. Let $S$ denote the set of points from the glued borders. It is also easily seen that there is a unique complex structure on $M$ compatible with those on $\Omega^{+}$and $\Omega^{-}$. If now $T \in \operatorname{Aut}(\Omega)$ then it extends to an automorphism of $M$ by the following formula which results from the Schwarz reflection principle:

$$
T^{*}(z)= \begin{cases}T(z) & \text { if } z \in \Omega^{+}, \\ \widetilde{T}(z) & \text { if } z \in S, \\ \overline{T(\bar{z})} & \text { if } z \in \Omega^{-} .\end{cases}
$$

where $\widetilde{T}$ is the homeomorphism from Theorem 2.3.

Of course ord $T=$ ord $T^{*}$ but more important is the observation that $\nu\left(T^{*}\right) \geq 2 \nu(T)$. The number of fixed points is thus at least doubled since there are now fixed points in both glued copies of $\Omega$ and perhaps some new ones on $S$.

Our main result in this part is now a simple consequence of the above observations.

THEOREM 2.4. If $\Omega$ is a p-connected bounded domain in the complex plane and $T \in \operatorname{Aut}(\Omega)$ has at least 2 fixed points, then ord $T \leq p$.

P r o of. First by Theorem 2.2 we can assume that $\Omega$ is a circular domain. Next, to estimate the order of $T$ it is enough to assume that $\Omega$ is proper. Thus we can consider the complex double $M$ and its automorphism $T^{*}$ defined above. As $M$ is a compact Riemann surface we can define $M^{*}$ to be the quotient space $M /\left\langle T^{*}\right\rangle$ and apply the Riemann-Hurwitz formula to the quotient map $\pi: M \rightarrow M^{*}$. Let $\gamma$ be as usual the genus of $M^{*}$ and $\mathrm{B}$ the branch index of $\pi$. As $T^{*}$ has at least 4 fixed points and the branch index of $\pi$ at each of them is $N-1$ where $N$ is the order of $T$, we get $B \geq 4(N-1)$ and hence $2 g-2 \geq N(2 \gamma-2)+4(N-1)$. Since $\gamma \geq 0$ it follows that $N \leq g+1=p$.

Remark 2.5. The assumption that $\Omega$ is bounded may be dropped; it suffices to assume that $\mathbb{C} \backslash \Omega$ has non-empty interior. In fact, if we consider 
domains on the Riemann sphere we can map any such domain by a Möbius transformation $\phi$ in such a way that the point at infinity is in the interior of $\widehat{\mathbb{C}} \backslash \phi(\Omega)$.

Remark 2.6. The result of Theorem 2.4 is sharp, that is, for every $p$ there exist a $p$-connected domain $\Omega$ and $T \in \operatorname{Aut}(\Omega)$ such that $\operatorname{ord} T=p$.

If $p=1$ then the domain is conformally equivalent to the unit disc and of course only its identity has two fixed points.

EXAMPLE 2.7. Let $p \geq 2$, and let $\varepsilon_{p}$ be a $p$-primitive root of 1 . Let $\delta$ be so small that the closed discs $D\left(\varepsilon_{p}^{r}, \delta\right), r=1, \ldots, p$, are pairwise disjoint. Then $\Omega=\widehat{\mathbb{C}} \backslash\left(D\left(\varepsilon_{p}^{1}, \delta\right) \cup \ldots \cup D\left(\varepsilon_{p}^{p}, \delta\right)\right)$ is a $p$-connected domain on the Riemann sphere. $T: \Omega \ni z \rightarrow \varepsilon_{p} z \in \Omega$ is its automorphism fixing 0 and the point at infinity and of course ord $T=p$.

Remark 2.8. Our paper does not deal with open Riemann surfaces at large but there are some straightforward conclusions. Namely, if we consider open Riemann surfaces of finite type (see Minda [M]) Theorem 1.4 still holds. For arbitrary open surfaces it seems that there is no good parameter to describe the order of an automorphism with two fixed points, and we must be satisfied with the result of Theorem 0.1 .

\section{References}

[B-S] H. Behnke und F. Sommer, Theorie der analytischen Funktionen einer komplexen Veränderlichen, Springer, Berlin 1976.

[F-K] H. M. F arkas and I. Kra, Riemann Surfaces, Graduate Texts in Math., Springer, New York 1980

[G] G. M. Goluzin, Geometric Theory of Functions of One Complex Variable, Gos. Izdat. Tekhn.-Teoret. Liter., Moscow 1952 (in Russian).

[H] W. J. Harvey, Cyclic groups of automorphisms of a compact Riemann surface, Quart. J. Math. Oxford Ser. (2) 17 (1966), 86-97.

[J-T] M. Jarnicki and P. Tworzewski, A note on holomorphic mappings with two fixed points, Ann. Polon. Math. 51 (1990), 179-182.

$[\mathrm{M}]$ E. Minda, Fixed points of analytic self-mappings of Riemann surfaces, Manuscripta Math. 27 (1979), 391-399.

INSTITUTE OF MATHEMATICS

JAGIELLONIAN UNIVERSITY

REYMONTA 4

30-059 KRAKÓW, POLAND 